\title{
Low-cost wake-up receiver for RF energy harvesting wireless sensor networks
}

K. Kaushik, Deepak Mishra, Swades De, Kaushik Roy Chowdhury and Wendi Heinzelman

The self-archived postprint version of this journal article is available at Linköping University Institutional Repository (DiVA):

http:// urn.kb.se/ resolve?urn=urn:nbn:se:liu:diva- 155753

N.B.: When citing this work, cite the original publication.

Kaushik, K., Mishra, D., De, S., Chowdhury, K. R., Heinzelman, W., (2016), Low-cost wake-up receiver for RF energy harvesting wireless sensor networks, IEEE Sensors J ournal, 16(16), 6270-6278.

https:/ / doi.org/ 10.1109/J SEN.2016.2574798

Original publication available at:

https://doi.org/ 10.1109/J SEN.2016.2574798

Copyright: Institute of Electrical and Electronics Engineers (IEEE)

http:// www.ieee.org/index.html

(C)2016 IEEE. Personal use of this material is permitted. However, permission to reprint/republish this material for advertising or promotional purposes or for creating new collective works for resale or redistribution to servers or lists, or to reuse any copyrighted component of this work in other works must be obtained from the IEEE. 


\title{
Low-cost Wake-up Receiver for RF Energy Harvesting Wireless Sensor Networks
}

\author{
K Kaushik, Deepak Mishra, Swades De, Kaushik Roy Chowdhury, and Wendi Heinzelman
}

\begin{abstract}
Wake-up receiver (WuRx) is a promising hardware solution that extends the life of a sensor node by switching off its radio to reduce idle listening energy consumption. The existing passive WuRxs are RFID tag based, which incur high cost and complexity. In this paper, we study cost-effective and long range WuRx solutions for range-based wake-up (RW) as well as directed wake-up (DW). In particular, we consider the case of an RF energy harvesting wireless sensor node and investigate how a low-cost WuRx can be built using an RF energy harvester available at the node without compromising the harvesting sensitivity and efficiency. Experimental results show that our developed prototype can achieve a wake-up range of 1.16 m with $+13 \mathbf{~ d B m}$ transmit power. Further, our empirical study shows that at $+30 \mathrm{dBm}$ transmit power the wake-up distance of our developed RW module is $>9 \mathrm{~m}$. High accuracy of DW is demonstrated by sending a 5-bit ID from a transmitter at a bit rate up to 33.33 kbps. Finally, we present optimized WuRx designs for RW and DW using Agilent advanced design system, which offer up to 5.69 times higher wake-up range for RW and energy savings per bit of about $0.41 \mathrm{~mJ}$ and $21.40 \mathrm{~nJ}$, respectively, at the transmitter and the sensor node in DW.
\end{abstract}

Index Terms-RF energy harvesting, passive wake-up receiver, range-based wake-up, directed wake-up, wireless sensor network

\section{INTRODUCTION}

Lifetime of a wireless sensor node is constrained by the energy present in its battery. Recharging through dedicated RF energy transfer [2], [3] using an RF energy harvester (RFEH) can enable its perpetual operation. However, in order to avoid frequent recharging, the battery energy should be judiciously used. Software solutions such as duty-cycled MAC protocols [4] use the energy wisely by keeping the node mostly in deep-sleep state. Yet, the nodes suffer from idle listening and overhearing, leading to increased energy consumption. These problems can be alleviated by using a wake-up receiver (WuRx) based solution [5]. A WuRx-enabled node can switch off its main radio and stay in deep-sleep state unless it needs to sample the sensor or is woken up by another node using a wake-up signal. Thus, a WuRx complements an RFEH in extending the node lifetime. Thanks to the presence of an

Preliminary results of this work were presented at IEEE Sensors Conference 2015 [1]: http://ieeexplore.ieee.org/stamp/stamp.jsp?tp=\&arnumber=7370562

K. Kaushik, D. Mishra, and S. De are with the Department of Electrical Engineering and Bharti School of Telecom, Indian Institute of Technology Delhi, New Delhi 110016, India (e-mail: \{eez128322, eez122429, swadesd\}@ee.iitd.ac.in).

K.R. Chowdhury is with the Department of Electrical and Computer Engineering, Northeastern University, Boston, MA 02115, USA (e-mail: krc@ece.neu.edu).

W. Heinzelman is with the Department of Electrical and Computer Engineering, University of Rochester, Rochester, NY 14623, USA (e-mail: wheinzel@ece.rochester.edu).
RFEH on the node in an RF energy harvesting wireless sensor network (WSN), a low-cost WuRx can be built with a few changes to the existing resources of the node.

\section{A. Prior art}

$R F$ energy harvesters: An RFEH converts the RF power available at its antenna to DC power [6]. An ideal RFEH is capable of harvesting RF energy simultaneously from multiple $\mathrm{RF}$ sources operating in different frequency bands with $100 \%$ RF-to-DC conversion efficiency [7]. However, various factors, such as, non-zero turn-on voltage of the diodes used for rectification, losses due to improper matching, and leakage current of the rectification diodes, are the bottlenecks in achieving full conversion efficiency [8].

In terms of reception sensitivity, commercially available RFEHs, such as Powercast P1110 [9], require a minimum input RF power of about $-11 \mathrm{dBm}$. This requirement is quite a bit higher than in most state-of-the-art prototypes [6], [8]. For example, the prototype in [8] was demonstrated to operate at input RF power as low as $-20 \mathrm{dBm}$. Further advances in the field of RFEHs has led to the development of energy harvesters that can harvest energy simultaneously from multiple RFbands [7] and use adaptive control techniques to achieve high conversion efficiency at low input RF powers [6], [10].

Wake-up receivers: Depending on the energy source, a WuRx can be broadly classified as: active - that draws energy from the node's battery for receiving a wake-up signal, and passive - that derives energy from the wake-up signal. The wake-up technique can be categorized into range-based wakeup $(R W)$ - where all the nodes wake up upon receiving a wake-up signal, and directed wake-up $(D W)$ - where a specific node is woken up by ID match [5]. An ideal WuRx should be passive, have high reception sensitivity and large wakeup range, and be capable of both $R W$ and $D W$. In [11], a radio triggered circuit based passive WuRx was introduced for RW, where it was shown through SPICE simulations that the wake-up range can be up to 30 meters with a 55 ms delay. DW function using multiple transceivers was also presented, where a node is woken up upon reception of a particular set of frequencies. However, hardware implementation based performance studies were not conducted in that work.

Another passive wake-up radio, called WISP-mote, was proposed in [12], where passive WuRx was demonstrated using Intel wireless identification and sensing platform (WISP). The wake-up range was shown to be 4 meters. The computing capabilities of the MSP430F2132 micro-controller $(\mu \mathrm{C})$ in WISP were used for ID decoding in DW. Later, it was experimentally demonstrated in [13] that the wake-up range 
of WISP-mote can be increased by using an additional RFEH. The authors in [13] also presented a passive WuRx design, called REACH-mote, that uses a reset circuit [14] along with an RFEH [8]. It was shown that, REACH-mote can provide a wake-up range of about 7.92 meters when using a Powercast transmitter with an effective isotropic transmit power (EIRP) of $3 \mathrm{~W}$ [9]. However, REACH-mote cannot perform DW.

Recently, a long-range passive WuRx design for RW was proposed in [15] by using an additional DC-DC converter. The authors also performed DW of an IRIS mote connected to a Monza X-2K passive RFID tag IC using its write wake-up. Though this design can achieve a large wake-up range, the DC-DC converter incurs significant hardware cost.

\section{B. Motivation}

As discussed above, most of the existing methods for node wake-up involves an overhead, either in terms of extra energy consumption or additional hardware. For example, a single pulse providing an input voltage greater than a threshold voltage $V_{\min }$ at the interrupt pin is sufficient for RW instead of using multiple pulses generated by a reset circuit [14] as shown in [13]. However, we note that an RFEH providing an output DC power at a certain voltage $V_{\text {out }}^{\mathrm{DC}}$, can act as a potential interrupt source when $V_{\text {out }}^{\mathrm{DC}} \geq V_{\min }$. Therefore by directly connecting the output of an RFEH to the interrupt pin of the $\mu \mathrm{C}$, one could not only obtain the same functionality provided by the reset circuit, but also reduce the current consumed by the reset circuit and obtain a sensor node with a small form factor. In this way, a single RFEH can be used for both operations, namely, energy harvesting and RW.

To the best of our knowledge, there is no existing device that performs both RW and DW using the same hardware and provides as long a wake-up range in DW as in RW. With the help of RFEH-based WuRx, we are able to demonstrate a different paradigm of hybrid wake-up where DW occurs by performing multiple RW in a given time window using the same RFEH and ultra-low power $\mu \mathrm{C}$ of the sensor node.

\section{Scope and contributions}

In this work, we first develop a hardware prototype of the WuRx and experimentally evaluate its performance. In order to have a compact design, we use the same antenna for RF energy harvesting as well as wake-up. Besides implementing a passive WuRx for RW, we develop an active WuRx design for DW. Since the WuRx is developed using an in-node RFEH, the achievable wake-up range and delay are limited by the underlying RFEH characteristics [16]. As an extension to our RFEH-based WuRx design, we also present two other solutions, which are independent of the node's RFEH.

In contrast to [13], our proposed WuRx does not require any reset circuit for its operation. Beyond the solution in [15], our module can perform both RW and DW with no changes to the hardware, where the type of wake-up is decided by the node's software and modulation of the wake-up transmitter (WuTx). Highlights and key contributions of this work are:

i) A low-cost RFEH-based WuRx design is proposed for supporting both wake-up and energy harvesting functions.

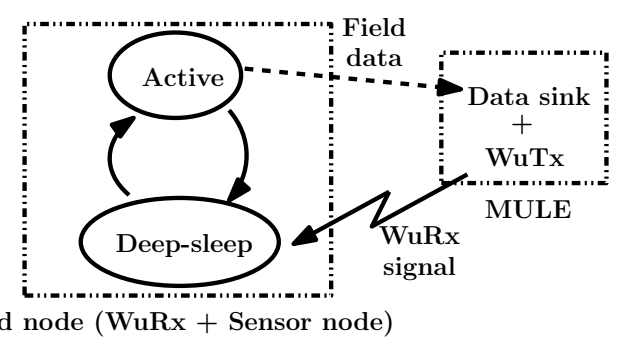

Fig. 1: Field sensor node wake-up (RW or DW) state diagram.

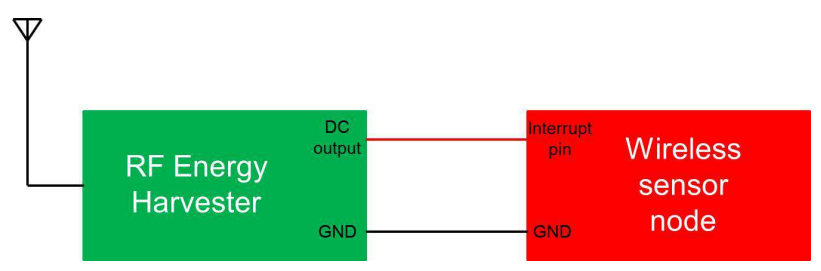

Fig. 2: Proposed simplified wake-up circuit.

ii) Implementation of RW provides insights on wake-up range at different values of transmit power.

iii) A protocol for decoding the ID in DW is developed. The accuracy of DW to selectively waking up a node using a 5-bit code ID is evaluated from hardware experiments for different values of bit duration.

iv) An empirical model is developed for characterizing the wake-up range versus transmit power for RW and DW.

v) Optimized WuRx designs independent of the underlying RFEH are proposed for efficient RW and DW.

\section{Paper organization}

The paper layout is as follows. Section II describes our proposed WuRx hardware prototype. Experimental evaluations of RW and DW using the developed prototype are presented respectively in Sections III and IV. Section V contains the empirical modeling of wake-up range. Design and simulationbased performance of our proposed optimal wake-up circuits are presented in Section VI. Section VII concludes the paper.

\section{Proposed RFEH-BASEd WuRx Design}

A delay-tolerant WSN comprised of a mobile ubiquitous LAN extension (MULE) for collecting data from static field sensor nodes is considered [3]. In order to save energy in the field sensor nodes, they are programmed to remain normally in deep-sleep state. For field data collection, the MULE sends a wake-up signal (RW or DW) to activate the node. After the data transfer, the node goes back to the deep-sleep state until it receives another wake-up signal. Fig. 1 shows the radio communications and the sensor node's state transition.

The block diagram of our proposed RFEH-based WuRx is shown in Fig. 2. It consists of an RFEH whose DC output is connected to the interrupt pin of the $\mu \mathrm{C}$ in the wireless sensor node. Unlike in the REACH-mote [13], our proposed WuRx module does not require a reset circuit. As will be shown in Section IV, the same prototype can also perform directed wake-up, which was not possible with the REACHmote approach. We next present a discussion on the choice of RFEH that we have considered throughout this paper. 


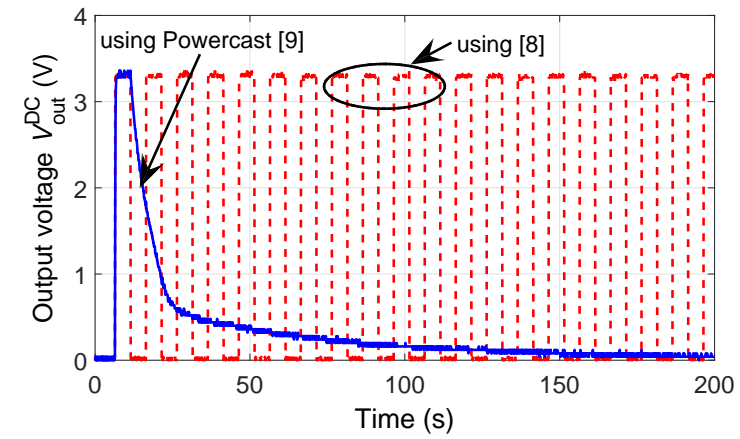

Fig. 3: Responsiveness to RF signal input in [8] and [9].

\section{A. Attributes of RFEH}

Two main features of WuRx are its wake-up range and wake-up delay. In our case, as the RFEH also acts as a WuRx, the attributes, namely, sensitivity and responsiveness of the WuRx can be mapped to those of the RFEH. In particular, an RFEH with high sensitivity and fast responsiveness acts as a WuRx with long wake-up range and small wake-up delay, respectively. Although any RFEH operating with low RF energy can be used, in our initial experimental and empirical evaluations we chose the 7-stage RFEH design presented in [8], which was originally designed as an efficient alternative to Powercast RFEH [9] in terms of higher reception sensitivity and RF-to-DC conversion efficiency. The RFEH in [8] is based on a Dickson voltage multiplier circuit, built using HSMS2852 Schottky diodes that have low turn-on voltage of $0.15 \mathrm{~V}$. As the RFEH is tuned to operate at a frequency of $915 \mathrm{MHz}$, our proposed WuRx also operates at this frequency.

\section{B. Comparison of RFEH in [8] with Powercast P1110 [9]}

We have conducted experiments to find the responsiveness of the RFEH in [8] and that of the Powercast P1110. To this end, an RFEH was allowed to harvest energy transmitted by an $\mathrm{RF}$ source in alternate 5 second periods using ON-OFF keying (OOK) modulation. Fig. 3 shows that the design in [8] is highly responsive to the ON and OFF transitions, as indicated by the small rise and fall times (on the order of a few tens of $\mu \mathrm{s}$ ) of output DC voltage $V_{\text {out }}^{\mathrm{DC}}$. Hence it can be used for DW, where the RF source (WuTx) sends OOK modulated ID of the sensor node as the wake-up signal. In contrast, although Powercast P1110 RFEH has small rise time, its fall time is on the order of a few tens of seconds, which is due to a large capacitor in the output stage of Powercast P1110 RFEH.

\section{Prototype of RFEH-based WuRx}

The prototype of our proposed RFEH-based WuRx enabled sensor node is shown in Fig. 4. The sensor node is comprised of a Texas Instruments (TI) eZ430-RF2500T having a 16bit ultra-low power $\mu \mathrm{C}$ TI MSP430F2274 and a $2.4 \mathrm{GHz}$ transceiver CC2500. The SMA connector on the RFEH is used to connect an antenna that operates at $915 \mathrm{MHz}$. Hereafter, the sensor node connected to the RFEH is termed as end node. Although the prototype has an RFEH, this harvester could support the operation of a node only in sleep state when it

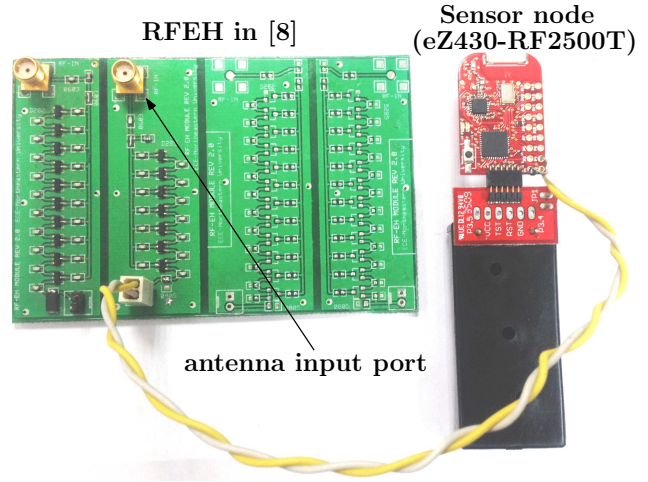

Fig. 4: Prototype of RFEH-based WuRx enabled sensor node.

draws a few $\mu \mathrm{A}$, but not in the active state when the node consumes about $270 \mu \mathrm{A} / \mathrm{MHz}$ at $2.2 \mathrm{~V}$. Due to this, the sensor node derives its energy for its regular sensing and common operations from an on-board battery, while the RFEH is used for passive wake-up. Next we present the RW experiments and the results of such a WuRx using this prototype.

\section{RANGE-BASED WAKE-UP}

Within the safe operation limits of the $\mu \mathrm{C}$ in a sensor node, $V_{\text {out }}^{\mathrm{DC}}$ of an RFEH can be categorized as LOW or HIGH corresponding to the $\mu \mathrm{C}$ 's low or high logic levels. Thus, the input RF power above a minimum threshold $P_{\mathrm{r} \text {,min }}$ causes its $V_{\text {out }}^{\mathrm{DC}}$ to go from LOW to HIGH. In other words, this transition of DC output voltage from LOW to HIGH generates a positive edge trigger, which interrupts the sleeping mote's $\mu \mathrm{C}$ to transit to the active state. Note that a simple voltage limiter such as a Zener diode is placed at the output of the RFEH to protect the sensor node's $\mu \mathrm{C}$ from potentially high voltage of the RFEH.

\section{A. Implementation}

By default, the node is kept in a deep-sleep state by switching off the radio completely and programming the MSP430F2274 $\mu \mathrm{C}$ to stay in low power mode 3 (LPM3). The wake-up signal received by the WuRx triggers the $\mu \mathrm{C}$ to transit from LPM3 to active state. After that, the mote's radio is switched on and a wake-up response (which may include field data) is sent to the WuTx. After the communication, the end node returns to deep-sleep state. For our experimental study, an Agilent N9310A RF source connected to a Powercast 6.1 $\mathrm{dBi}$ antenna (acting as WuTx of the MULE) was used to send a wake-up signal at $915 \mathrm{MHz}$. Another $6.1 \mathrm{dBi}$ Powercast antenna connected to the RFEH (cf. Fig. 4) was used as the prototype WuRx. Throughout the experiments, to find the maximum wake-up range, both the WuTx and WuRx were placed on a line of sight at a height of $80 \mathrm{~cm}$ above the floor.

\section{B. Experimental results for $R W$}

Experiments were limited to short-range communication because the maximum transmit signal power of the RF transmitter used in our experiments is $+13 \mathrm{dBm}$. The maximum wake-up range that can be achieved at different RF transmit power levels using our prototype is indicated by the solid curve in Fig. 5. This figure shows that the wake-up range of the prot- 


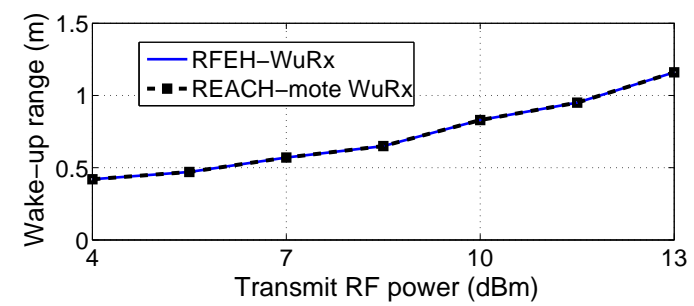

Fig. 5: Effect of transmit power on WuRx range.

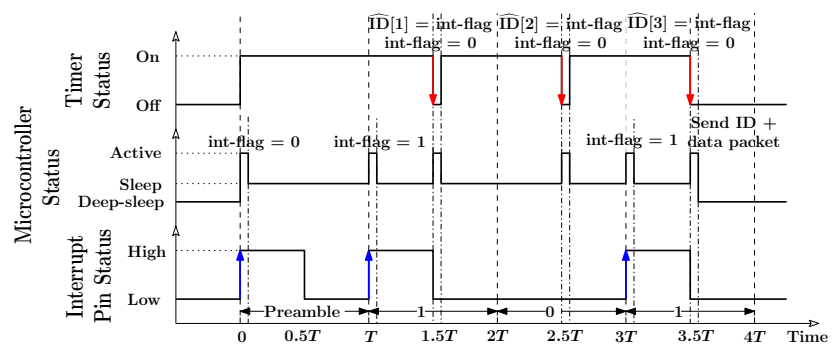

Fig. 6: Directed wake-up timing diagram for ID $=101$.

otype WuRx at $+13 \mathrm{dBm}$ transmit power is $1.16 \mathrm{~m}$. Fig. 5 also shows that the wake-up range of the prototype is identical to that of the REACH-mote [13]. It may be recalled that, with respect to the REACH-mote design, the proposed prototype is more cost-effective as it eliminates the need for a reset circuit.

\section{DiRected WAKE-UP}

We now present how DW is performed using our developed prototype. Through the experiments we also show how the decoding accuracy depends on bit duration of the ID sequence.

\section{A. Implementation}

The main idea behind DW using our proposed RFEH-based WuRx prototype is: an incoming $R F$ signal generates a sudden increase in harvested energy at the output of the RFEH. That is, $V_{\text {out }}^{\mathrm{DC}}$ sharply transits from LOW-to-HIGH upon reception of an RF signal. Also, $V_{\text {out }}^{\mathrm{DC}}$ goes from HIGH-to-LOW shortly after removal of the RF signal input. This implies that, a suitably-modulated RF wake-up signal may be used to wakeup an intended node. After identifying a series of interruptions within a fixed time, the $\mu \mathrm{C}$ can interpret or decode the target node ID. In Fig. 6, we present a timing diagram for the steps involved in decoding the DW ID '101', with duration of each bit as $T$ s. For transmission of the intended ID, we use:

- $O O K$ modulation - to generate an interrupt at the $\mu \mathrm{C}$ upon receiving a ' 1 ' bit, none upon receiving a ' 0 ' bit.

- $R Z$ encoding - to enable detection of consecutive ' 1 ' bits in an RF WuTx signal that is modulated by a WuRx ID.

- 1-bit preamble (' 1 ') - to ensure that the $\mathrm{WuRx}$ is able to decode even a binary code starting with a ' 0 ' bit.

A detailed explanation of the RFEH-based DW procedure, involving reception and decoding, is provided in Algorithm 1.

\section{B. Experimental study}

Different component blocks of the static field node and MULE for DW operation are shown in Fig. 7(a). We have

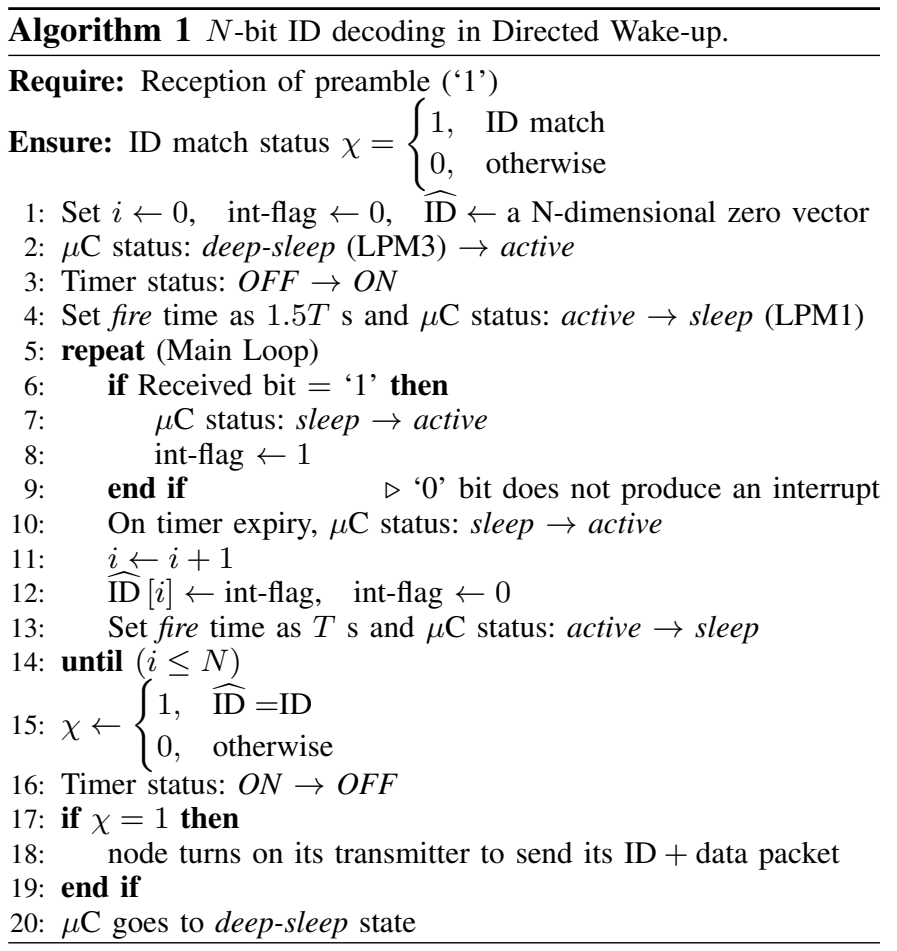

performed hardware experiments to investigate decoding accuracy of the proposed RFEH-based DW at varying bit duration. For this, WuTx of the MULE and WuRx of the end node were placed at a height of $80 \mathrm{~cm}$ above the floor, separated by 80 $\mathrm{cm}$. The experimental setup is shown in Fig. 7(b), and the component specifications are listed in Table I.

TABLE I: System specifications.

\begin{tabular}{ll}
\hline \hline Device & Specification \\
\hline Data sink & eZ430-RF2500T + eZ430-RF USB \\
RF source & Agilent N9310A RF signal generator \\
& transmitting at 915 MHz \\
RZ waveform generator & Agilent 33220A arbitrary waveform generator \\
RFEH & 7-stage voltage multiplier [8] \\
Sensor node & eZ430-RF2500T + 2 AAA batteries \\
\hline \hline
\end{tabular}

For experimental evaluation of DW, an end node was assigned an $N=5$ bit node ID '11111'. On reception of an $N+1$ OOK modulated ' 111111 ' wake-up signal (which includes a 1-bit ('1') preamble) from the MULE and subsequent ID match, the end node sends an ACK to the data sink, which is stored as a record in a laptop. This process was repeated $10^{4}$ times, with the subsequent record of matching stored in the laptop to evaluate the DW success rate.

\section{Experimental results for $D W$}

Since DW is carried out by performing multiple RW within a fixed time using the same RFEH, the maximum wakeup range for DW is identical to that of RW. Thus, with our available maximum $+13 \mathrm{dBm}$ WuTx power and $6.1 \mathrm{dBi}$ antenna gains, the DW range is about $1.16 \mathrm{~m}$ (cf. Fig. 5).

To study the effect of transmission rate on successful wakeup, we varied the bit duration from $10 \mu \mathrm{s}$ to $40 \mu \mathrm{s}$. For each bit duration, the RZ waveform generator sends the 6 bit DW 


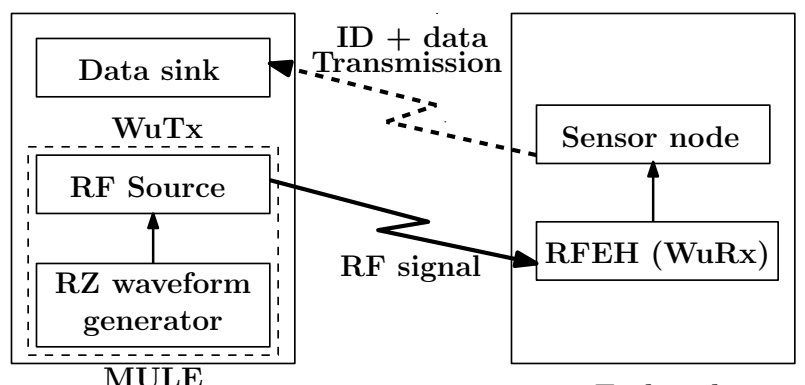

(WuTx + Data sink)

(a) Block diagram of DW and data collection process

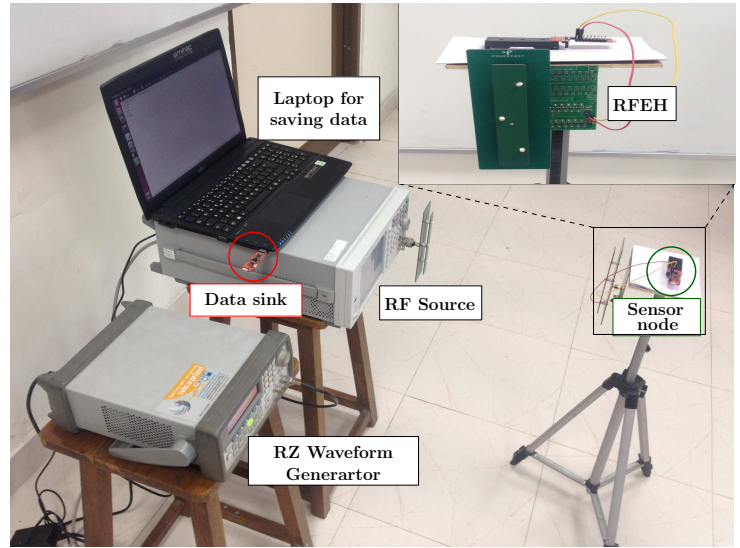

(b) Experimental setup for DW

Fig. 7: Directed wake-up.

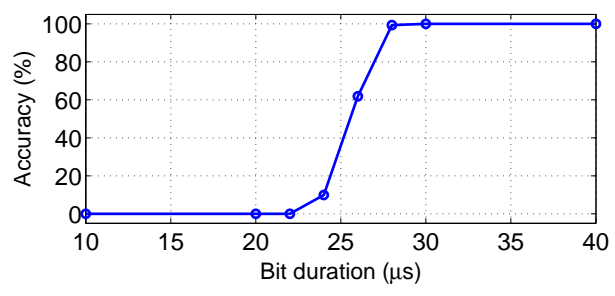

Fig. 8: Decoding accuracy versus bit duration.

signal $10^{4}$ times, with a guard time between the adjacent signals. This guard time is to ensure that the end node is able to receive the DW signal and send back an ACK packet to the data sink. Decoding accuracy for various values of bit duration is shown in Fig. 8. Since the considered RZ code in the 5-bit sequence '11111' contains the highest number of transitions, this bit stream represents the worst-case scenario of bit errors. Hence, from the plot it can be concluded that a 5-bit DW signal transmitted with a bit rate of up to $33.33 \mathrm{kbps}(30 \mu \mathrm{s}$ bit duration) can be decoded with $100 \%$ accuracy. This bit rate is higher than the achieved rate in some of the recent WuRx techniques. For example, the approach in [17] offers successful wake-up at a bit rate of 0.5 to $8 \mathrm{kbps}$.

\section{EMPIRICAL MOdel FOR Estimating WAKe-UP RANGE}

In this section, based on experimental observations of the wake-up range in RW and DW, we develop an empirical model that provides an estimate of the maximum achievable wake-up range with the proposed RFEH-based WuRx design.

\section{A. Variation of $V_{\min }$ with supply voltage of mote}

The possibility of a RW follows the idea that an interrupt causes the on-board $\mu \mathrm{C}$ to transit from sleep state to active state. The minimum voltage $V_{\min }$ needed for the interrupt to occur depends on the supply voltage $V_{\mathrm{cc}}$ of the $\mu \mathrm{C}$ on the sensor node. As the pins of a $\mu \mathrm{C}$ contain a Schmitt trigger to avoid input signal noise [18], $V_{\min }$ is same as positive going input threshold voltage [19]. We have performed experiments to find the dependence of $V_{\min }$ on supply voltage $V_{\text {mote }}$ of the mote. Also, the $\mu \mathrm{C}$ on the TI mote (eZ430-RF2500T) is directly powered by the battery. Therefore, $V_{\mathrm{cc}}=V_{\text {mote }}$. We

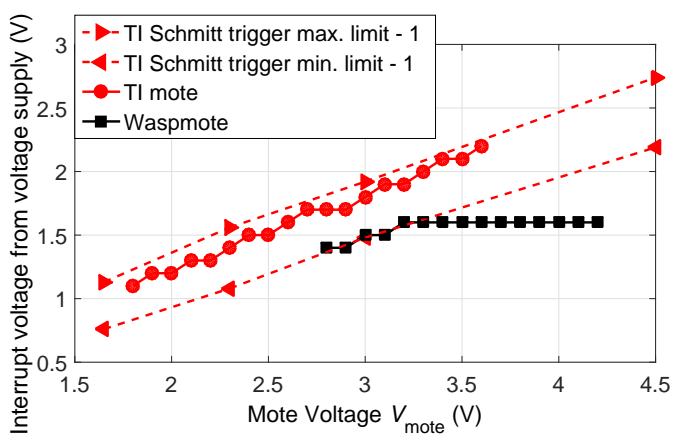

Fig. 9: Minimum voltage for triggering various motes.

have emulated the wake-up process by connecting a voltage supply to the sensor node to power the $\mu \mathrm{C}$, and another voltage supply acts as a source for the external interrupts. In order to capture the behavior of motes in general, we have done experiments using a TI mote [20] as well as a Waspmote [21].

Fig. 9 confirms that $V_{\min }$ of the TI mote for various values of $V_{\text {mote }}$ is between the maximum and minimum levels of the positive going input threshold voltage of the TI Schmitt trigger [19]. As the Atmega1281 $\mu \mathrm{C}$ present on the Waspmote uses a $3.3 \mathrm{~V}$ low drop-out regulator [21], $V_{\min }$ of the $\mu \mathrm{C}$ is held constant at $3.3 \mathrm{~V}$ even when $V_{\text {mote }}$ is in the safe operating range of $3.3 \mathrm{~V}$ to $4.2 \mathrm{~V}$. Due to this, $V_{\min }$ for the Waspmote is constant for $V_{\text {mote }}>3.3 \mathrm{~V}$. To gain analytical insights on the performance of RW, we develop equations (1) and (2) by curve fitting the experimental results for minimum interrupt voltage $V_{\min }^{\mathrm{TI}}$ and $V_{\min }^{\mathrm{W}}$ for TI mote and the Waspmote, respectively.

$$
\begin{gathered}
V_{\min }^{\mathrm{TI}} \approx 0.5965 V_{\text {mote }}+0.0316,, \forall 1.8 \mathrm{~V} \leq V_{\text {mote }} \leq 3.6 \mathrm{~V} . \\
V_{\min }^{\mathrm{W}} \approx \begin{cases}0.4 V_{\text {mote }}+0.27, & 2.8 \mathrm{~V} \leq V_{\text {mote }}<3.2 \mathrm{~V} \\
1.6 \mathrm{~V}, & 3.2 \mathrm{~V} \leq V_{\text {mote }} \leq 4.2 \mathrm{~V}\end{cases}
\end{gathered}
$$

\section{B. Current consumption comparison of $R W$ scheme}

Another set of experiments have been performed to find the current consumed by the interrupt pin $\left(I_{\text {int }}\right)$. Current measurements for the TI mote using a Keithley 6485 picoammeter indicates that the current drawn is $<10 \mathrm{nA}$ at the instant of interrupt arrival. For the rest of time, current consumed is almost zero. We have noted that, as the interrupt 


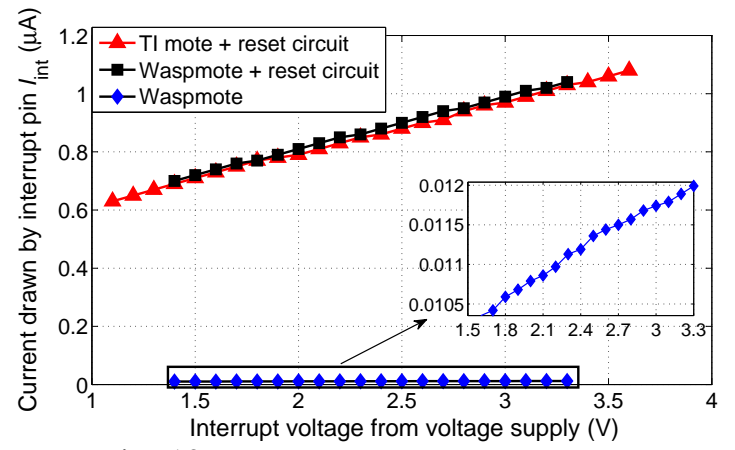

Fig. 10: Current consumed by interrupt pin.

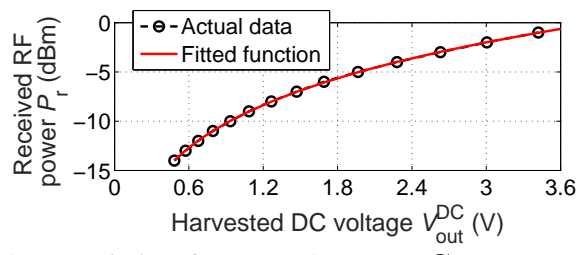

Fig. 11: Characteristic of our used RFEH. $C_{\text {stage }}=36 \mathrm{pF}, L=68$ $\mathrm{nH}, C=3.3 \mathrm{pF}, R=220 \mathrm{M} \Omega$ (cf. Fig. 13)

pin that we have used on the Waspmote is also used for alternative functions, it continuously draws a current in the range of $10 \mathrm{nA}$ to $13 \mathrm{nA}$ (shown in Fig. 10). We have also measured the current consumed by the reset circuit [13] that is used in the REACH mote-based WuRx approach. Fig. 10 shows that, though the current consumed by the reset circuit is in the sub-micro ampere range, it is about 100 times more than that consumed directly by the interrupt pin. Thus, with our our approach the interrupt pin does not load the RFEH.

\section{Relationship between wake-up range and transmit power}

The relationship between received RF power and output DC voltage of RFEH [8] (Fig. 11), derived from experiments, is:

$$
P_{r}(\mathrm{~mW})=0.0486\left(V_{\text {out }}^{\mathrm{DC}}\right)^{2}+0.0659 V_{\text {out }}^{\mathrm{DC}}-0.0031 .
$$

Fig. 9 shows that the minimum required $V_{\text {mote }}$ for operating the TI mote and the Waspmote are respectively $1.8 \mathrm{~V}$ and $2.8 \mathrm{~V}$. Following the discussion in Section $\mathrm{V}-\mathrm{A}, P_{\mathrm{r}, \mathrm{min}}$ for the TI mote and the Waspmote for these minimum $V_{\text {mote }}$ values are determined by substituting $V_{\min }^{\mathrm{TI}}=1.1 \mathrm{~V}$ and $V_{\text {min }}^{\mathrm{W}}=1.4 \mathrm{~V}$ for $V_{\mathrm{out}}^{\mathrm{DC}}$ in equation (3) as: $\left\{P_{\mathrm{r}, \mathrm{min}}^{\mathrm{TI}}, P_{\mathrm{r}, \mathrm{min}}^{\mathrm{W}}\right\}=$ $\{-8.9213,-7.3420\} \mathrm{dBm}$. Further, on using the Friis transmission equation, the wake-up range can be estimated as:

$$
\text { Range }(\mathrm{m})=\lambda\left(P_{\mathrm{t}} G_{\mathrm{t}} G_{\mathrm{r}}\right)^{\frac{1}{n}}(4 \pi)^{-1} P_{\mathrm{r}}^{-\frac{1}{n}}
$$

where $n$ is the path loss exponent, $\lambda$ is the operating wavelength, $P_{\mathrm{t}}, P_{\mathrm{r}}$ and $G_{\mathrm{t}}, G_{\mathrm{r}}$ represent, respectively, the transmit power, receive power and gains of the transmitter and receiver antennae. Considering the parameter values as $G_{\mathrm{t}}=6.1 \mathrm{dBi}$, $G_{\mathrm{r}}=6.1 \mathrm{dBi}, n=2$, and $\lambda=0.328 \mathrm{~m}$, the maximum wakeup range that can be obtained by our RFEH-WuRx prototype at various values of input power and mote voltage $V_{\text {mote }}$ is shown in Fig. 12. For a fixed RF source transmit power, the wake-up range increases with reduction in the required $V_{\text {mote }}$. It shows that with the considered parameters, for transmit power of 1

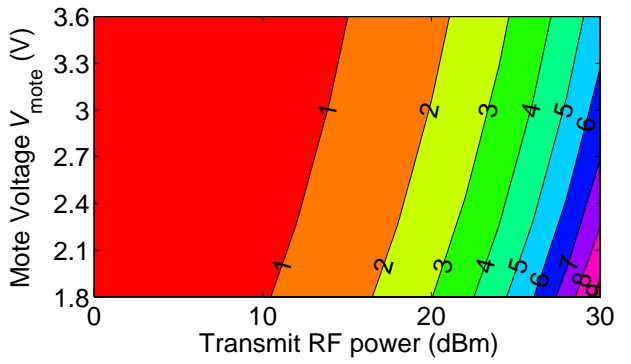

Fig. 12: Wake-up range (in $\mathrm{m}$ ), indicated by numbers in the graph.

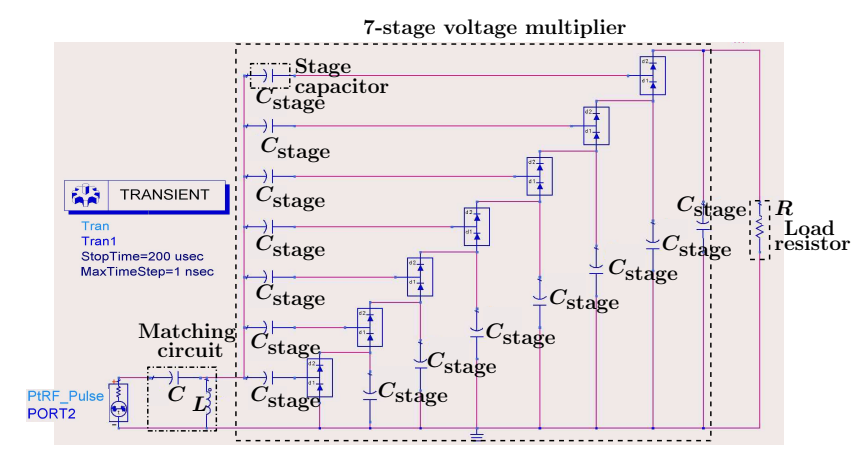

Fig. 13: ADS simulation block diagram of RFEH using a 7-stage Dickson voltage multiplier.

$\mathrm{W}$ (or EIRP of $4 \mathrm{~W}$ ), in the best case scenario $\left(V_{\text {mote }}=1.8 \mathrm{~V}\right.$ $\Rightarrow V_{\min }=1.1 \mathrm{~V}$, from Fig. 9), we obtain a wake-up range of more than $9 \mathrm{~m}$. Furthermore, with a $3 \mathrm{~W}$ EIRP Powercast transmitter [9], our achievable wake-up range of $8.06 \mathrm{~m}$ is marginally higher than $7.92 \mathrm{~m}$ range of REACH-mote [13].

\section{Optimized WuRX Design}

Until now, we have studied how an RFEH can be reused as a WuRx to perform both RW and DW. However, the operable bit rate and wake-up range are bottlenecked by the characteristics of the RFEH. In this section, via ADS simulation studies we design an optimized circuit solely for WuRx purposes that provides a much longer wake-up range for RW. We also show that an optimally-designed circuit solely for DW operation can support ID decoding at a higher bit rate and provide greater energy savings. These independent circuits trade-off cost to some extent for better WuRx characteristics, because a separate RFEH would be required for optimal energy harvesting. Specifically, in the optimized WuRx circuit design, without being concerned about RF harvesting efficiency, we intend to improve the wake-up performance by analyzing the effect of all parameters, namely, stage capacitor $C_{\text {stage, }}$, number of stages, load resistor $R$, and matching circuit (capacitor $C$, inductor $L$ ) (cf. Fig. 13). Since $V_{\text {mote }}=3.6 \mathrm{~V}$ gives the wakeup range for the worst case scenario for a given transmit RF power (cf. Fig. 12), we consider $V_{\min }=2.2 \mathrm{~V}$ that corresponds to $V_{\text {mote }}=3.6 \mathrm{~V}$ (cf. Fig. 9). By dividing $V_{\text {out }}=V_{\text {min }}=2.2 \mathrm{~V}$ with the current consumed by the interrupt pin of the $\mu \mathrm{C}$ (= $10 \mathrm{nA}$ ) (cf. Section V-B), the load $R$ in the RFEH is found to be $220 \mathrm{M} \Omega$. Unless otherwise stated, we consider $C_{\text {stage }}=36 \mathrm{pF}, R=220 \mathrm{M} \Omega, C=3.3 \mathrm{pF}$, and $L=68 \mathrm{nH}$ for generating simulation results. Next we discuss the impact and optimization of $C_{\text {stage }}, C, L$ and the number of stages. 


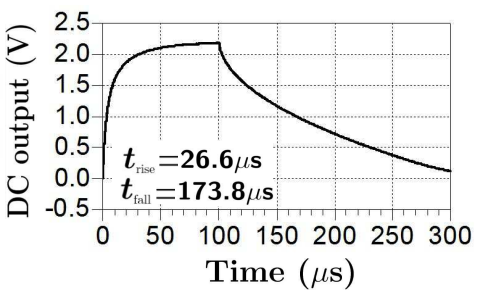

(a) $C_{\text {stage }}=36 \mathrm{pF}[8]$

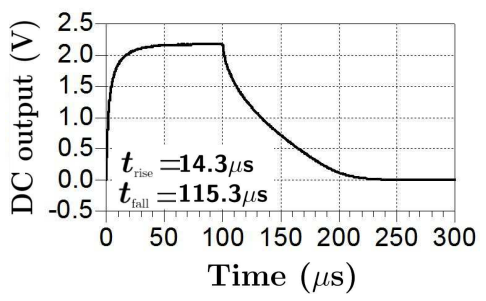

(b) $C_{\text {stage }}=18 \mathrm{pF}$

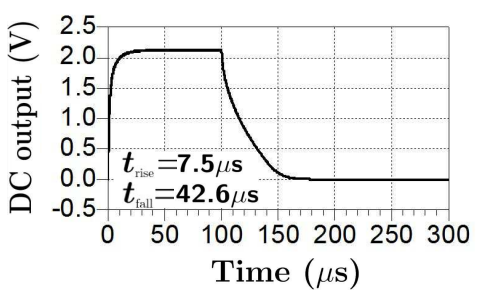

(c) $C_{\text {stage }}=9 \mathrm{pF}$

Fig. 14: Effect of $C_{\text {stage }}$ value on $t_{\text {rise }}$ and $t_{\text {fall }} . R=220 \mathrm{M} \Omega, C=3.3 \mathrm{pF}, L=68 \mathrm{nH}$, stages $=7, P_{\mathrm{r}, \min }=-3.4 \mathrm{dBm}$ for $V_{\text {out }}^{\mathrm{DC}}=2.2 \mathrm{~V}$.

\section{A. Effect of stage capacitor $C_{\text {stage }}$}

Since $C_{\text {stage }}$ determines the sharpness of wake-up response, which primarily affects the pulse width and hence bit rate in DW, optimum $C_{\text {stage }}$ is decided within the DW context.

Energy saving in DW is achieved if the $\mu \mathrm{C}$ remains in deepsleep state (LPM3) for a longer period ${ }^{1}$. This can be achieved by reducing the time taken by the $\mu \mathrm{C}$ for ID decoding. The key idea is to reduce the rise time and fall time for a bit reception at the WuRx, which helps reduce the bit-duration, thus enabling faster ID decoding and thereby saving energy. As the stage capacitors store energy before delivering it to the load, the $C_{\text {stage }}$ value highly affects the rise and fall times. In order to compare the optimized 7-stage design for WuRx with the 7-stage RFEH [8] used in our experiments (which was originally designed and optimized for charging a supercapacitor of a field node), we have conducted simulations.

We have performed transient simulation using Agilent ADS (block diagram shown in Fig. 13) to study the rise time $\left(t_{\text {rise }}\right)$ and fall time $\left(t_{\text {fall }}\right)$ of an RFEH by subjecting it to an RF signal pulse of duration $100 \mu$ s. Simulation results on $V_{\text {out }}^{\mathrm{DC}}$ in Fig. 14 show the effect of $C_{\text {stage }}$ value on rise/fall times. The actual values of $t_{\text {rise }}$ and $t_{\text {fall }}$ for all $C_{\text {stage }}$ values $(36 \mathrm{pF}, 18 \mathrm{pF}, 9$ $\mathrm{pF})$ are also obtained. ( $t_{\text {rise }}$ is calculated as the time taken for the DC output to rise from $10 \%$ to $90 \%$ of the steady-state value. Similarly, $t_{\text {fall }}$ is the time taken for the DC output to fall from $90 \%$ to $10 \%$ of the steady-state level). Reduction of stage capacitance value beyond $9 \mathrm{pF}$ introduces instability in steady-state response. Note that a single bit duration is mainly comprised of $t_{\text {rise }}, t_{\text {fall }}$ and interrupt detection time $\left(t_{\text {detect }}\right)$. As $t_{\text {detect }}$ is low (sub- $\mu$ s to a few $\mu$ s [18]) compared to $t_{\text {rise }}$ and $t_{\text {fall }},\left(t_{\text {rise }}+t_{\text {fall }}\right)$ contribute to the majority of bit duration. With $C_{\text {stage }}=9 \mathrm{pF}, t_{\text {rise }}+t_{\text {fall }}$ reduces to one-fourth of the duration with $C_{\text {stage }}=36 \mathrm{pF}$. This leads to about four times reduction in bit duration. Thus, a WuRx with $C_{\text {stage }}=9 \mathrm{pF}$, when used for DW, quadruples the operable bit rate compared to that with $C_{\text {stage }}=36 \mathrm{pF}$. As the energy consumption in DW is proportional to the decoding time, a reduction in bit duration results in energy savings (Section VI-C). The same optimum stage capacitor value is used in the optimized WuRx for RW.

\section{B. Effect of matching circuit $(C, L)$ and number of stages}

The load resistance $R$, matching circuit $(C, L)$, and number of voltage multiplier stages in the RFEH in combination affect the wake-up range for a given transmitted RF power and

${ }^{1}$ For TI mote with $V_{\text {mote }}=3 \mathrm{~V}$, the measured power consumption in LPM3 and sleep state (LPM1) are $0.3 \mu \mathrm{W}$ and $261.3 \mu \mathrm{W}$, respectively.

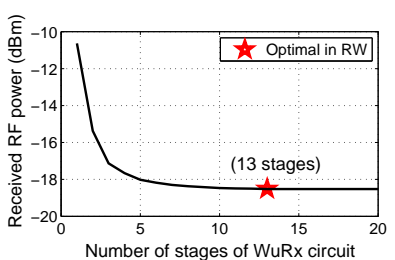

Fig. 15: Effect of stages. $C_{\text {stage }}=9 \mathrm{pF}, R=220 \mathrm{M} \Omega$

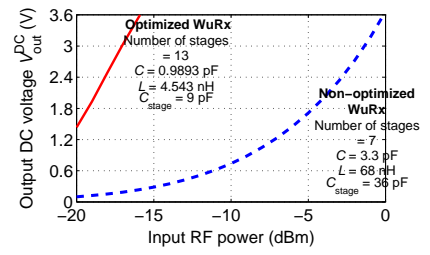

Fig. 16: Response of different WuRx circuits for RW.

the required $V_{\text {out }}^{\mathrm{DC}}$ threshold. Therefore, optimization of these parameters are discussed in the context of RW.

Based on the observations from Fig. 14 and related discussion, we set $C_{\text {stage }}=9 \mathrm{pF}$ and $R=220 \mathrm{M} \Omega$ to find the optimal number of stages for WuRx. Harmonic balance analysis is used to find the optimum matching parameters ( $C=0.9893 \mathrm{pF}$ and $L=4.543 \mathrm{nH}$ ), that provide the maximum DC output for RF input power in the range of -20 $\mathrm{dBm}$ to $0 \mathrm{dBm}$. RF input power $P_{\mathrm{r} \text {,min }}$ required for $V_{\mathrm{out}}^{\mathrm{DC}}=2.2$ $\mathrm{V}$ with varying number of stages is plotted in Fig. 15. It shows that $P_{\mathrm{r}, \mathrm{min}}$ with increased number of stages gets saturated beyond 13-stages. So we conclude that 13-stage circuit design is optimal as it helps in achieving long wake-up range.

In Fig. 16 we plot the variation of $V_{\text {out }}^{\mathrm{DC}}$ with $\mathrm{RF}$ input power for optimized WuRx design parameters $\left(C_{\text {stage }}=9 \mathrm{pF}\right.$, $L=4.543 \mathrm{nH}, C=0.9893 \mathrm{pF}$, and number of stages $=13$ ) and non-optimized parameters $\left(C_{\text {stage }}=36 \mathrm{pF}, C=3.3\right.$ $\mathrm{pF}, L=68 \mathrm{nH}$, and number of stages $=7$ ). We observe that the optimized design achieves significant improvement in wake-up performance over the RFEH-based WuRx design (cf. Section II). Fig. 16 shows that the optimized WuRx requires $P_{\mathrm{r}, \mathrm{min}}^{\mathrm{TI}}=-18.52 \mathrm{dBm}(=0.01406 \mathrm{~mW})$ for a minimum interrupt voltage $V_{\min }=2.2 \mathrm{~V}$. In contrast, with non-optimized RFEH-based WuRx requires $P_{\mathrm{r} \text {,min }}^{\mathrm{TI}}=-3.41$ $\mathrm{dBm}(=0.4560 \mathrm{~mW})$ for $V_{\min }=2.2 \mathrm{~V}$, which is significantly higher. It may be noted that output DC voltage is clipped at $3.6 \mathrm{~V}$ with the help of a Zener diode to avoid damage to the $\mu \mathrm{C}$. Thus, by optimizing the RFEH parameters there is a saving of about $96.92 \%\left(=\frac{0.4560-0.01406}{0.4560} \times 100 \%\right)$ in the required RF power with optimized design, for achieving the same wake-up range as in the non-optimized design. For a Powercast transmitter with EIRP $\left(P_{r} G_{\mathrm{t}}\right)=3 \mathrm{~W}, \lambda=0.328$ $\mathrm{m}$, and considering $G_{\mathrm{r}}=6.1 \mathrm{dBi}$, and $n=2$, equation (4) gives the maximum wake-up range that can be achieved by 13-stage independent WuRx design in the worst-case scenario $\left(V_{\min }=2.2 \mathrm{~V}\right)$ as $24.41 \mathrm{~m}$, which is 5.69 times the wake-up range achieved by RFEH-based WuRx design $(=4.29 \mathrm{~m})$. 
TABLE II: Effect of number of stages with optimized WuRx for DW. $R=220 \mathrm{M} \Omega, C_{\text {stage }}=9 \mathrm{pF}$

\begin{tabular}{cccccc}
\hline \hline No. of stages & $\boldsymbol{C}(\mathbf{p F})$ & $\boldsymbol{L}(\mathbf{n H})$ & $\boldsymbol{t}_{\text {rise }}(\boldsymbol{\mu s})$ & $\boldsymbol{t}_{\text {fall }}(\boldsymbol{\mu s})$ & $\boldsymbol{P}_{\mathbf{r , m i n}}(\mathbf{d B m})$ \\
\hline 1 & 0.2832 & 46.310 & 0.5 & 8.0 & -10.625 \\
2 & 0.3647 & 26.540 & 3.5 & 13.5 & -15.380 \\
3 & 0.4608 & 18.260 & 16.2 & 22.5 & -17.0357 \\
4 & 0.5372 & 13.990 & 32.3 & 27.9 & -17.667 \\
5 & 0.5967 & 11.370 & 33.2 & 30.9 & -18.000 \\
6 & 0.6487 & 9.589 & 45.6 & 37.4 & -18.1667 \\
7 & 0.6965 & 8.287 & 55.6 & 43.4 & -18.2917 \\
\hline \hline
\end{tabular}

\section{Wake-up speed versus range tradeoff in $D W$}

While the load resistance $(R=220 \mathrm{M} \Omega)$ and its corresponding optimum matching circuit parameters remain valid for DW as well, an increased number of voltage multiplier stages reduces the wake-up speed $\left(\propto \frac{1}{t_{\text {rise }}+t_{\text {fall }}}\right)$ while increasing the wake-up range. Therefore, in this section we study the optimum number of voltage multiplier stages as a tradeoff between the wake-up speed and the wake-up range.

As shown in Fig. 17, wake-up delay $\left(t_{\text {rise }}+t_{\text {fall }}\right)$ monotonically increases with the number of stages due the presence of a large number of stage capacitors. In order to save energy both at the WuRx and the WuTx, we further optimize the WuRx circuit to not only operate at a higher bit rate but also consume lower power in the wake-up process. For this, we first perform harmonic balance simulations to find the optimum matching circuit parameters and then conduct transient simulations for each of the designs to find rise and fall times. Table II shows the optimum matching parameters $(C, L), t_{\text {rise }}, t_{\text {fall }}$ for varying number of stages with $C_{\text {stage }}=9 \mathrm{pF}$ and $R=220 \mathrm{M} \Omega$. $P_{\mathrm{r} \text {,min }}$ denotes the minimum input power corresponding to a steadystate DC output value of $2.2 \mathrm{~V}$, which is the same as $V_{\min }^{\mathrm{TI}}$ for $V_{\text {mote }}=3.6 \mathrm{~V}$. Although the capacitor values shown in Table II are low, they are realizable in practice [22].

It is important to note that, due to non-optimized matching circuit parameters of the 7-stage RFEH used in the hardware experiments, $P_{\mathrm{r}, \min }=-4.25 \mathrm{dBm}$ for generating an output voltage $V_{\text {out }}=2.2 \mathrm{~V}$ (from Fig. 11). On the other hand, optimized 7-stage $\mathrm{WuRx}$ design requires $P_{\mathrm{r}, \min }=-18.2917 \mathrm{dBm}$ for $V_{\text {out }}=2.2 \mathrm{~V}$. Therefore, the optimized 7-stage design produces significantly large wake-up range compared to the non-optimized design. To make a fair comparison on the achievable bit rate, we consider the result shown in Fig. 14(c) and the result from 7-stage design in Table II (because of having the same $C_{\text {stage }}=9 \mathrm{pF}$ and number of stages $=7$ ). Instead of comparing $t_{\text {rise }}+t_{\text {fall }}$ in both cases, let us observe $t_{\text {rise }}$ and $t_{\text {fall }}$ separately. Since the input power is very low for the optimized 7-stage WuRx design $(=-18.2917 \mathrm{dBm})$, it takes more time $(55.6 \mu \mathrm{s}$, from Table II) to rise to the steady state value of $2.2 \mathrm{~V}$. On the other hand, with the nonoptimized circuit, it is significantly lower $(7.5 \mu \mathrm{s})$, which is due to the high input power. From this we conclude that $t_{\text {rise }}$ is also affected by matching circuit parameters. Once the circuit reaches a steady-state value of $2.2 \mathrm{~V}, t_{\text {fall }}$ is more or less constant in both cases (cf. Fig. 14(c) and Table II).

Fig. 17 plots the trade-off between the rise/fall times and the achievable wake-up range obtained using equation (4) with a Powercast transmitter EIRP $\left(P_{\mathrm{t}} G_{\mathrm{t}}\right)=3 \mathrm{~W}, G_{\mathrm{r}}=6.1 \mathrm{dBi}$, $n=2, \lambda=0.328 \mathrm{~m}$ and $P_{\mathrm{r}}$ as the minimum input power

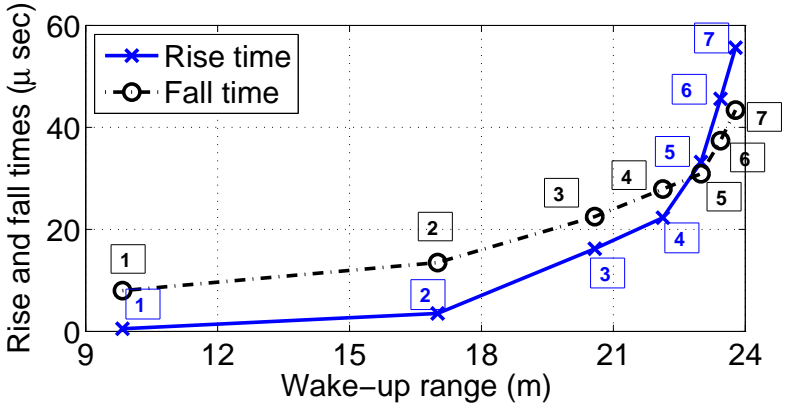

Fig. 17: Trade-off between rise/fall time and wake-up range in DW. Numbers in squared box indicate the number of stages (cf. Table II for optimum matching parameters $(C, L)$ and $\left.P_{\mathrm{r}, \min }\right)$.

mentioned in Table II for varying number of stages from 1 to 7. Results plotted show that the optimal number of stages in DW depends upon the specific requirement of having high wake-up speed or high wake-up range. It is observed that, due to the wake-up speed versus wake-up range tradeoff in DW, the optimal number stages is two because with a higher number of stages, the rise and fall times increase sharply without a significant improvement in wake-up range. Lower rise time and fall time with a 2-stage design as compared to a 7-stage design provides significant energy savings.

Energy saving per bit at the WuTx and WuRx can be estimated by multiplying the respective power consumption with the difference in $t_{\text {rise }}+t_{\text {fall }}$ in 7-stage design versus 2stage design (which is $99.0-17.0=82 \mu \mathrm{s}$, from Table II). For a $3 \mathrm{~W}$ EIRP Powercast transmitter, the power consumption is $5 \mathrm{~W}$ [9]. So the energy saving per bit in DW at the WuTx is $0.41 \mathrm{~mJ}$. From the timing diagram of DW in Fig. 6, power consumption at the $\mu \mathrm{C}$ is $0.261 \mathrm{~mW}(3 \mathrm{~V} \times 87.1 \mu \mathrm{A})$ in LPM1 (sleep) state. Hence, the energy saving per bit in DW at the WuRx is $21.40 \mathrm{~nJ}$. Overall, the optimized energy saving in the optimized 2-stage design over 7 -stage design is $82.82 \%$.

\section{CONCLUding REMARKS}

We have proposed a low-cost RFEH-based WuRx design capable of performing both RW and DW. Hardware implementation results have demonstrated that the proposed prototype can achieve a long wake-up range in RW and high accuracy in DW at a bit rate up to $33.33 \mathrm{kbps}$. Empirical results-assisted analytical modeling of wake-up range has shown that a node at a distance greater than $9 \mathrm{~m}$ from the WuTx can be woken up using either RW or DW. Further, using ADS simulations, we have studied the effect of RFEH parameters and proposed a 13-stage RFEH-based independent WuRx design with the optimized parameters for long range wake-up in RW. It has been estimated that using a $3 \mathrm{~W}$ EIRP Powercast transmitter, compared to a non-optimized design, an optimized design can increase the wake-up range by up to 5.69 times. We have also proposed an independent WuRx design for DW that is optimized for higher range as well as higher energy savings at both WuTx and WuRx (sensor node). Balancing the trade-off between rise/ fall time and wake-up range, it has been shown that a 2-stage design is optimal in case of DW. This optimized design can provide energy saving per bit of about $0.41 \mathrm{~mJ}$ and $21.40 \mathrm{~nJ}$, respectively, at the WuTx and WuRx. 
It may be noted that an independent WuRx solution requires a separate antenna for its operation in addition to the RFEH circuit and an associated antenna for recharging the node's battery, which incurs some additional cost. However the advantage is that, this approach allows the WuRx to flexibly operate on a different frequency other than the RFEH frequency. Further, independence of the WuRx operation from the RFEH suggests that this solution can be used in other WSNs.

\section{ACKNOWLEDGMENT}

This work was supported jointly by the Department of Electronics and Information Technology (DeitY) and the National Science Foundation (NSF) awards Green Sensor Networks for Air Quality Support (grant numbers DeitY 13(2)/2012CC\&BT, NSF CNS 1143662) and NSF CNS 1452628.

\section{REFERENCES}

[1] K. Kaushik et al., "RF energy harvester-based wake-up receiver," in Proc. IEEE Sensors, Busan, Korea, Nov. 2015.

[2] S. De and R. Singhal, "Toward uninterrupted operation of wireless sensor networks," IEEE Computer Mag., vol. 45, no. 9, pp. 24-30, Sep. 2012.

[3] D. Mishra et al., "Smart RF energy harvesting communications: Challenges and opportunities," IEEE Commun. Mag., vol. 53, no. 4, pp. 70-78, Apr. 2015.

[4] J. Oller et al., "Has time come to switch from duty-cycled mac protocols to wake-up radio for wireless sensor networks?" IEEE/ACM Trans. Netw., vol. 24, no. 2, pp. 674-687, Apr. 2016.

[5] I. Demirkol, C. Ersoy, and E. Onur, "Wake-up receivers for wireless sensor networks: Benefits and challenges," IEEE Wireless Commun., vol. 16, no. 4, pp. 88-96, Aug. 2009.

[6] Z. Hameed and K. Moez, "A $3.2 \mathrm{~V}-15 \mathrm{dBm}$ adaptive thresholdvoltage compensated RF energy harvester in $130 \mathrm{~nm}$ CMOS," IEEE Trans. Circuits Syst. I, vol. 62, no. 4, pp. 948-956, Apr. 2015.

[7] V. Kuhn et al., "A multi-band stacked RF energy harvester with RF-toDC efficiency up to 84\%," IEEE Trans. Microw. Theory Techn., vol. 63, no. 5, pp. 1768-1778, May 2015.

[8] P. Nintanavongsa et al., "Design optimization and implementation for RF energy harvesting circuits," IEEE J. Emerg. Sel. Topics Circuits Syst., vol. 2, no. 1, pp. 24-33, Mar. 2012.

[9] Powercast corp. wireless power solutions. [Online]. Available: http://www.powercastco.com/

[10] P.-H. Hsieh, C.-H. Chou, and T. Chiang, "An RF energy harvester with $44.1 \%$ PCE at input available power of $-12 \mathrm{dBm}$," IEEE Trans. Circuits Syst. I, vol. 62, no. 6, pp. 1528-1537, Jun. 2015.

[11] L. Gu and J. A. Stankovic, "Radio-triggered wake-up for wireless sensor networks," Real-Time Syst., vol. 29, no. 2-3, pp. 157-182, Mar. 2005.

[12] H. Ba, I. Demirkol, and W. Heinzelman, "Feasibility and benefits of passive RFID wake-up radios for wireless sensor networks," in Proc. IEEE GLOBECOM, Miami, FL, USA, Dec. 2010.

[13] L. Chen et al., "Range extension of passive wake-up radio systems through energy harvesting," in Proc. IEEE ICC, Budapest, Hungary, Jun. 2013, pp. 1549-1554.

[14] P. Simpson, "Ultra low power reset generator," Maxim Integrated, Tech. Rep. 1186, Sep. 2002.

[15] D. De Donno, L. Catarinucci, and L. Tarricone, "Ultralong-range RFIDbased wake-up radios for wireless sensor networks," IEEE Sensors J., vol. 14, no. 11, pp. 4016-4017, Nov. 2014.

[16] D. Mishra et al., "Implementation of multi-path energy routing," in Proc. IEEE PIMRC, Washington D.C., USA, Sep. 2014, pp. 1834-1839.

[17] J. Oller et al., "Performance evaluation and comparative analysis of subcarrier modulation wake-up radio systems for energy-efficient wireless sensor networks," Sensors, vol. 14, no. 1, pp. 22-51, 2013.

[18] Texas Instruments MSP430F2274 Micro-controller. [Online]. Available: http://www.ti.com/product/msp430f2274

[19] C. Cockrill, "Understanding schmitt triggers," Texas Instruments, Application report SCEA046, Sep. 2011.

[20] MSP430 wireless development tool. [Online]. Available: http://www.ti.com/tool/ez430-rf2500

[21] Libelium [Online]. Available: http://www.libelium.com/products/waspmote

[22] Mouser Electronics. [Online]. Available: http://www.mouser.in/PassiveComponents/Capacitors/_/N-5g7r/

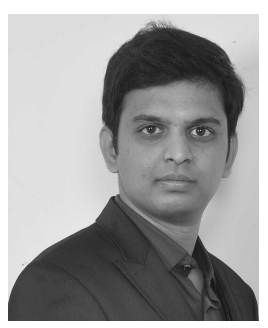

K Kaushik received his M.E. degree in Communication Engineering from Birla Institute of Technology and Science, Pilani, Rajasthan, India, in 2012. He is currently pursuing the Ph.D. degree from the Department of Electrical Engineering, IIT Delhi, India. His research interests include energy harvesting wireless sensor networks and embedded systems.

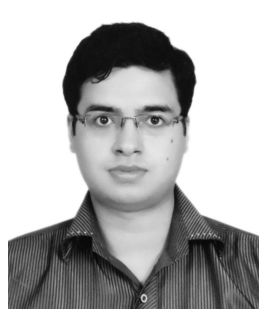

Deepak Mishra (S'13) received his B.Tech degree in Electronics and Communication Engineering from Guru Gobind Singh Indraprastha University, Delhi, India, in 2012. He is currently pursuing the Ph.D. degree from the Department of Electrical Engineering, IIT Delhi, India. His research interests include RF energy harvesting cooperative communication networks and energy optimization schemes for uninterrupted operation of mobile ad hoc networks.

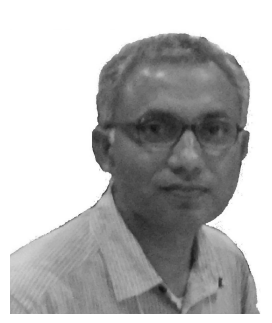

Swades De (S'02-M'04-SM'14) received the Ph.D. degree from the State University of New York at Buffalo, NY, USA, in 2004. He is currently an Associate Professor in the Department of Electrical Engineering, IIT Delhi, India. In 2004, he worked as an ERCIM researcher at ISTI-CNR, Italy. From 2004 to 2006 he was with NJIT as an Assistant Professor. His research interests include performance study, resource efficiency in wireless networks, broadband wireless access, and optical communication systems.

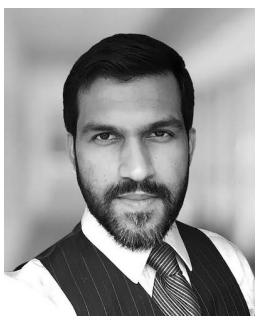

Kaushik Roy Chowdhury (M'09-SM'15) is Associate Professor in the Electrical and Computer Engineering Department at Northeastern University, with previous employment as Assistant Professor from 2009-2015 in the same university. He received his Ph.D. from the Georgia Institute of Technology in August 2009 and M.S. from the University of Cincinnati in 2006. He is the winner of the ONR Director of Research Early Career award in 2016 and the NSF CAREER award in 2015. He received the best paper award at the IEEE International Conference on Communications (ICC) in 2013, 2012 and 2009, as well as the best paper award at the International Conference on Computing, Networking and Communications (ICNC) in 2013. He is the current Chair of the IEEE Technical Committee on Simulation and Sr. Member of the IEEE. He serves as the area editor for the following journals: Elsevier Ad Hoc Networks, Elsevier Computer Communications, and EAI Transactions on Wireless Spectrum.

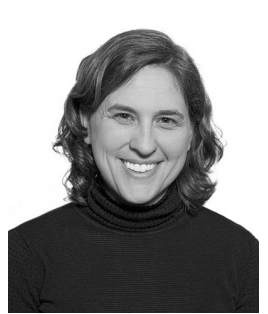

Wendi Heinzelman is a Professor in the Department of Electrical and Computer Engineering at the University of Rochester, with a secondary appointment in the Computer Science Department at Rochester. She also currently serves as the Dean of Graduate Studies for Arts, Sciences \& Engineering. Dr. Heinzelman received a B.S. degree in Electrical Engineering from Cornell University in 1995 and M.S. and Ph.D. degrees in Electrical Engineering and Computer Science from MIT in 1997 and 2000, respectively. Her current research interests lie in the area of wireless communications and networking, mobile computing, and multimedia communication. Dr. Heinzelman is a member of Networking Networking Women $\left(\mathrm{N}^{2}\right.$ Women) and the Society of Women Engineers (SWE), a Distinguished Scientist of ACM SIGMOBILE, and a Fellow of the IEEE Communications Society, the IEEE Signal Processing Society and the IEEE Computer Society. 